ARTICLE

\title{
Low-noise GaAs quantum dots for quantum photonics
}

\author{
Liang Zhai (1) ${ }^{1 凶}$, Matthias C. Löbl(10 ${ }^{1}$, Giang N. Nguyen ${ }^{1,2}$, Julian Ritzmann (1) ${ }^{2}$, Alisa Javadi (1) ${ }^{1}$, \\ Clemens Spinnler (1) ${ }^{1}$, Andreas D. Wieck (iD ${ }^{2}$, Arne Ludwig (i) ${ }^{2} \&$ Richard J. Warburton (iD ${ }^{1}$
}

Quantum dots are both excellent single-photon sources and hosts for single spins. This combination enables the deterministic generation of Raman-photons-bandwidth-matched to an atomic quantum-memory-and the generation of photon cluster states, a resource in quantum communication and measurement-based quantum computing. GaAs quantum dots in $\mathrm{AlGaAs}$ can be matched in frequency to a rubidium-based photon memory, and have potentially improved electron spin coherence compared to the widely used InGaAs quantum dots. However, their charge stability and optical linewidths are typically much worse than for their InGaAs counterparts. Here, we embed GaAs quantum dots into an n-i-p-diode specially designed for low-temperature operation. We demonstrate ultra-low noise behaviour: charge control via Coulomb blockade, close-to lifetime-limited linewidths, and no blinking. We observe high-fidelity optical electron-spin initialisation and long electron-spin lifetimes for these quantum dots. Our work establishes a materials platform for low-noise quantum photonics close to the red part of the spectrum.

\footnotetext{
${ }^{1}$ Department of Physics, University of Basel, Klingelbergstrasse 82, $\mathrm{CH}-4056$ Basel, Switzerland. ${ }^{2}$ Lehrstuhl für Angewandte Festkörperphysik, Ruhr-Universität Bochum, DE-44780 Bochum, Germany. ${ }_{\text {email: liang.zhai@unibas.ch }}$
} 
O uantum dots (QDs) in III-V semiconductors form excellent sources of indistinguishable single-photons. These emitters have a combination of metrics (brightness, purity, coherence, repetition rate) which no other source can match ${ }^{1-4}$. These excellent photonic properties can be extended by trapping a single electron to the QD, enabling spin-photon entanglement ${ }^{5}$ and high-rate remote spin-spin entanglement creation ${ }^{6}$. Underpinning these developments are, first, a selfassembly process to create nano-scale QDs; and second, a smart heterostructure design along with high-quality material. The established platform consists of InGaAs QDs embedded in GaAs. However, the InGaAs QDs emit at wavelengths between 900 and $1200 \mathrm{~nm}$, a spectral regime lying inconveniently between the telecom wavelengths $(1300 \mathrm{~nm}$ and $1550 \mathrm{~nm})$ and the wavelength where silicon detectors have a high efficiency ${ }^{7}(600-800 \mathrm{~nm})$. It is important in the development of QD quantum photonics to extend the wavelength range towards both, shorter and longer wavelengths.

GaAs QDs in an AlGaAs matrix can be self-assembled by local droplet etching 8,9 and have a spectrally narrow ensemble ${ }^{10,11}$. They emit at wavelengths between 700 and $800 \mathrm{~nm}$. This is an important band: it coincides with the peak quantum efficiency of silicon detectors; it contains the rubidium $\mathrm{D}_{1}$ and $\mathrm{D}_{2}$ wavelengths (795 $\mathrm{nm}$ and $780 \mathrm{~nm}$, respectively) offering a powerful route to combining QD photons with a rubidium-based quantum memory $^{12}$. Furthermore, GaAs QDs have typically more symmetric shapes, facilitating the creation of polarisation-entangled photon pairs from the biexciton cascade ${ }^{4,13}$.

GaAs QDs have also very low levels of strain ${ }^{9,14-17}$. In contrast, the high level of strain in InGaAs QDs complicates the interaction of an electron spin with the nuclear spins on account of the atomic site-specific quadrupolar interaction ${ }^{14,18}$. For electrostatically defined GaAs QDs, the spin-dephasing time, $T_{2}^{*}$, has been prolonged to the micro-second regime by narrowing the nuclear spin distribution together with real-time Hamiltonian estimation $^{19}$. Applied to a droplet GaAs QD, such techniques could prolong the spin dephasing time to values several orders of magnitude above the radiative lifetime. In this case, in combination with optical cavities ${ }^{20}$, droplet GaAs QDs can potentially serve as fast, high-fidelity sources of spin-photon pairs and cluster states ${ }^{21}$.

The development of GaAs QDs for quantum photonics lags far behind the InGaAs QDs. Recurrent problems are blinking ${ }^{22,23}$ (telegraph noise in the emission) and optical linewidths well above the transform limit ${ }^{13,16,23-25}$. Both of these problems are caused by charge noise. On short time-scales, the charge environment is static such that successively emitted photons exhibit a high degree of coherence ${ }^{4,25}$. On longer time-scales, however, the charge noise introduces via blinking an unacceptable stochastic character to the photon stream. An additional weak non-resonant laser provides control over the noise to a certain extend, though it does not remove the blinking completely ${ }^{22}$.

For InGaAs QDs, embedding the QDs in an $n$ - $i-p$ diode has profound advantages: the charge state is locked by Coulomb blockade ${ }^{26-28}$; the charge noise is reduced significantly ${ }^{29}$; and the exact transition frequency can be tuned in-situ via a gate voltage ${ }^{3,30}$. Such a structure is missing for GaAs QDs ${ }^{13,16,22-25}$ in previous attempts, charge-stability was not demonstrated ${ }^{31,32}$. A materials issue must be addressed: the barrier material AlGaAs must be doped, yet silicon-doped AlGaAs contains DXcentres $^{33,34}$ which both reduce the electron concentration, causing the material to freeze out at low temperatures, and lead to complicated behaviour under illumination. Here, we resolve this issue-all doped $\mathrm{AlGaAs}$ layers have a low $\mathrm{Al}$-concentration. In this case, the DX level lies above the conduction band minimum and thus is unoccupied at cryogenic temperatures ${ }^{33}$. The QDs are grown in a region with higher Al-concentration, which is wellestablished for the growth of these QDs ${ }^{8}$. On GaAs QDs in this device we demonstrate charge-control via Coulomb blockade, optical linewidths just marginally above the transform limit, blinking-free single-photon emission, electron spin initialisation, and a spin-relaxation time as large as $\sim 50 \mu \mathrm{s}$.

\section{Results}

Sample design and characterisation. The sample is grown on a GaAs-substrate with (001)-orientation. Below the active region of the sample, a distributed Bragg reflector is grown to enhance the collection efficiency of the photons emitted by the QDs. The QDs are embedded in an $n$-i-p-diode structure where the QDs are tunnel-coupled to the $n$-type layer. The $n$-type back gate consists of silicon-doped $\mathrm{Al}_{0.15} \mathrm{Ga}_{0.85}$ As. The low Al-concentration in this layer is crucial to avoid the occupation of DX-centres in $n$-type $\mathrm{AlGaAs}^{33,34}$. A tunnel barrier consisting of $20 \mathrm{~nm} \mathrm{Al}_{0.15} \mathrm{Ga}_{0.85} \mathrm{As}$ followed by $10 \mathrm{~nm} \mathrm{Al}_{0.33} \mathrm{Ga}_{0.67} \mathrm{As}$ separates the QDs from the $n$ type back gate. The QDs are grown in the $\mathrm{Al}_{0.33} \mathrm{Ga}_{0.67} \mathrm{As}$-layer by using local droplet-etching 8 . The QD-density is $n_{\mathrm{QD}}=0.37 \pm$ $0.01 \mu \mathrm{m}^{-2}$. Above the QDs, there is $274 \mathrm{~nm}$ of $\mathrm{Al}_{0.33} \mathrm{Ga}_{0.67} \mathrm{As}$ followed by a $p$-type top gate. The top gate is composed of carbon-doped $\mathrm{Al}_{0.15} \mathrm{Ga}_{0.85} \mathrm{As}$, where reduced Al-concentration is used as well. A schematic bandstructure of the diode is shown in Fig. 1a; all Al-concentrations in this design are small enough that processing into micropillars ${ }^{35}$ and nanostructures will not be hindered by oxidation ${ }^{36}$. In Table 1 , the design of the full heterostructure is given.

We characterise our device by measuring the photoluminescence from a single $\mathrm{QD}$ as a function of the gate voltage, $V_{\mathrm{g}}$, applied to the diode (Fig. 1b). As a function of $V_{\mathrm{g}}$, the emission lines show a pronounced Stark-shift. At specific gate voltages, discrete jumps in the emission spectrum take place: one emission line abruptly becomes weaker and another line appears. This effect is the characteristic signature of charge-control of a QD via Coulomb blockade ${ }^{26}$ : the net-charge of the QD increases one by one and the emission energy is shifted due to the additional Coulomb interaction with the new carrier.

We fit the relation $E=E_{0}+\alpha F+\beta F^{2}$ to the dependence of the emission energy, $E$, on electric field, $F$ (Supplementary Fig. 2). The energy jumps between different charge plateaus are removed for the fit. We find $\alpha / \mathrm{e}=0.21 \mathrm{~nm}$, the permanent dipole moment in the growth direction, and $\beta=-1.35 \times 10^{-6} \mathrm{eV}(\mathrm{kV} / \mathrm{cm})^{-2}$, the polarisability of the $\mathrm{QD}^{37}$. Extrapolating the fit shows that the Stark shift is zero at a non-zero electric field $\left(F=7.8 \mathrm{kVcm}^{-1}\right)$. The non-zero value of $\alpha$ represents a small displacement between the "centre-of-mass" of the electron and the hole wavefunctions. The hole wavefunction is slightly closer to the back gate than the electron wavefunction.

Resonance fluorescence from GaAs QDs. We identify the neutral exciton, $\mathrm{X}^{0}$, from its characteristic fine-structure splitting, as well as a quantum-beat in time-resolved resonance fluorescence (Supplementary Fig. 3). For our device, the fine-structure splittings are distributed over a range of $1-3 \mathrm{GHz}$ (see Supplementary Fig. 4c). The fine-structure splittings are comparable to literature values on (001)-oriented samples ${ }^{4,8}$. Smaller fine-structure splittings can be obtained by using (111)-oriented samples ${ }^{13}$ and strain-tuning ${ }^{38}$. We identify the other charge-states by counting the number of jumps in the emission spectrum as the gate-voltage increases/decreases. We measure emission from highly charged excitons ranging from the two-times positively charged exciton, $\mathrm{X}^{2+}$, to the eight-times negatively charged exciton, $\mathrm{X}^{8-}$. Such a wide range of charge tuning was not previously achieved with any QDs emitting in the close-to-visible wavelengths. Our GaAs QDs 
a

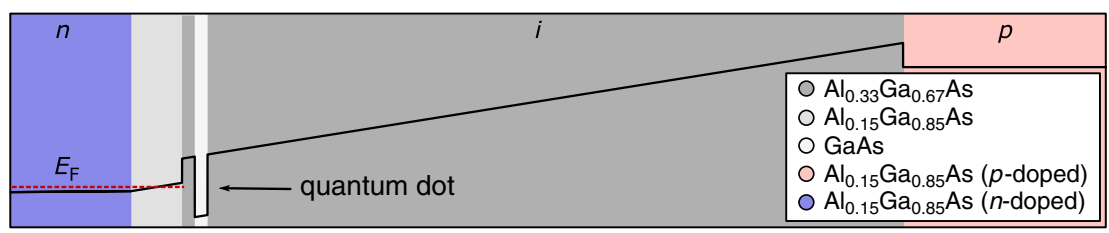

b

\begin{tabular}{|l|l|l|l|l|l|l|l|l|l|l|}
\hline $\mathrm{X}^{2+}$ & $\mathrm{X}^{1+}$ & $\mathrm{X}^{0}$ & $\mathrm{X}^{1-}$ & $\mathrm{X}^{2-}$ & $\mathrm{X}^{3-}$ & $\mathrm{X}^{4-}$ & $\mathrm{X}^{5-}$ & $\mathrm{X}^{6-}$ & $\mathrm{X}^{7-}$ & $\mathrm{X}^{8-}$ \\
\hline
\end{tabular}

$F\left(\mathrm{kV} \mathrm{cm}^{-1}\right)$

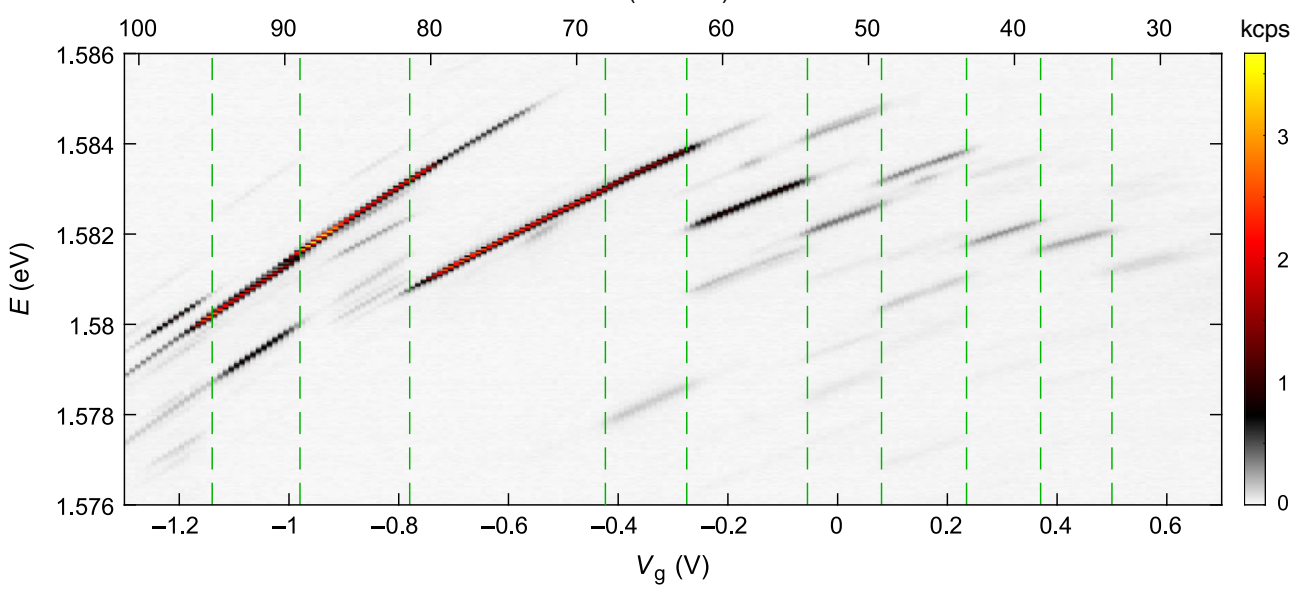

C

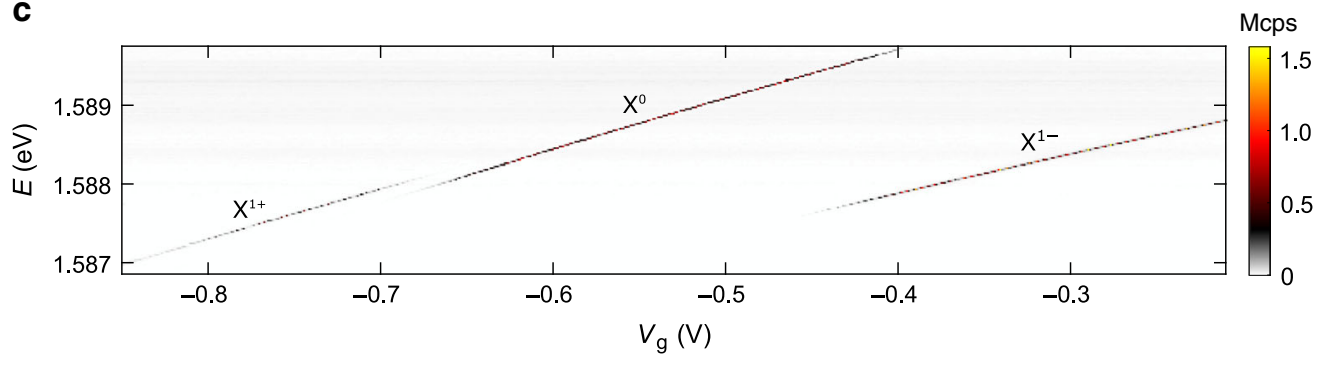

Fig. 1 Tuning the charge state of single GaAs quantum dots. a Schematic band structure (conduction band) of the diode hosting charge-tunable GaAs quantum dots. $\mathbf{b}$ The photoluminescence emitted by an exemplary single quantum dot as a function of the gate voltage, $V_{\mathrm{g}}$. (Positive gate voltage indicates a forward bias.) The corresponding electric field, $F$, is plotted as an additional $x$-axis on top. The photoluminescence is resolved in energy by a spectrometer and measured on a CCD-camera. The emission spectrum shows several plateaus corresponding to different charge states of the quantum dot. We observe narrow photoluminescence-linewidths on highly charged excitons where up to eight additional electrons occupy the quantum dot. c Resonance fluorescence from $X^{1+}, X^{0}$, and $X^{1-}$ charge plateaus measured on another quantum dot (QD1). $X^{1+}, X^{0}$, and $X^{1-}$ represent the positive trion, the neutral exciton, and the negative trion, respectively. The measurement is performed by sweeping the gate voltage for different laser frequencies. The resonance fluorescence intensity is measured with a superconducting nanowire single-photon detector. This measurement is performed by resonant continuous-wave excitation below saturation. In saturation, the maximum count rate is $6.5 \mathrm{MHz}$ (see Supplementary Fig. 1 for the power saturation curve).

give a large range of charge tuning due to their relatively large size $^{8}$ in comparison to the widely used InGaAs $\mathrm{QDs}^{39}$.

We turn to resonant excitation. This excitation scheme is key for creating low-noise photons and represents a true test of the fidelity of the device as, unlike photoluminescence, continuum states are not deliberately occupied. By sweeping both the gate voltage and excitation laser frequency, we map out three charge plateaus of a single quantum dot $(\mathrm{QD} 1)-\mathrm{X}^{1+}, \mathrm{X}^{0}$, and $\mathrm{X}^{1-}$ (see Supplementary Fig. 5 for photoluminescence of QD1). As is visible in Fig. 1c, the exact transition energy of all three charge states can be tuned via $V_{\mathrm{g}}$ across a range of above $1 \mathrm{meV}$. At a fixed gate voltage, we determine a resonance fluorescence linewidth of $\mathrm{X}^{1-}$ to be $0.64 \pm 0.01 \mathrm{GHz}$ (full width at half maximum) on scanning a narrow-bandwidth laser over the trion resonance (see Fig. 2a). (resonance fluorescence laser scans on $\mathrm{X}^{1+}$ and $\mathrm{X}^{0}$ are shown in Supplementary Fig. 3). This measurement takes several minutes: the linewidth probes the sum of all noise sources over an enormous frequency bandwidth $^{40}$. The measured linewidth is very close to the lifetime-limit of $\Gamma_{\mathrm{r}} / 2 \pi=0.59 \pm 0.01 \mathrm{GHz}$. (It is assumed here the decay is radiative. The radiative decay rate $\Gamma_{\mathrm{r}}$ is determined by recording a decay curve following pulsed resonant excitation, Fig. 2b). This result shows that there is extremely little linewidth broadening due to noise in our device. These excellent results are not limited to one individual QD. Shown in Fig. 2d is a linewidth measurement on a second QD (QD2). In the central part of the $\mathrm{X}^{1-}$ charge-plateau (from $V_{\mathrm{g}}=-0.5 \mathrm{~V}$ to $V_{\mathrm{g}}=-0.4 \mathrm{~V}$ in Fig. 2c), we also measure a close-to lifetime-limited linewidth. On average, the ratio between the measured linewidth and the lifetime limit is 1.08 for QD2. At the edges of the charge-plateau, the linewidth increases-a well-know effect due to a co-tunnelling interaction with the Fermi-reservoir ${ }^{41}$. Comparably good properties are found for in total seven out of ten randomly chosen QDs with $\mathrm{X}^{1-}$ below $785 \mathrm{~nm}$ (see Supplementary Fig. 4a, b). 
Table 1 Sample design with relevant growth parameters.

\begin{tabular}{|c|c|c|c|c|}
\hline Material & Thickness (nm) & Temperature $\left({ }^{\circ} \mathrm{C}\right)$ & Duration (s) & Comments \\
\hline GaAs:C & 5 & 540 & 25.1 & $\mathrm{p}^{++}$-doped epitaxial gate \\
\hline $\mathrm{Al}_{0.15} \mathrm{Ga}_{0.85} \mathrm{As}: \mathrm{C}$ & 10 & 540 & 42.7 & $\mathrm{p}^{++}$-doped epitaxial gate \\
\hline $\mathrm{Al}_{0.15} \mathrm{Ga}_{0.85} \mathrm{As}: \mathrm{C}$ & 65 & 540 & 277.7 & $\mathrm{p}^{+}$-doped epitaxial gate \\
\hline $\mathrm{Al}_{0.33} \mathrm{Ga}_{0.67} \mathrm{As}$ & 273.6 & 540 & 921.8 & Blocking barrier \\
\hline GaAs & 2 & 605 & 10 & Filling of the etched nano-holes \\
\hline- & - & 605 & 60 & Droplet etching \\
\hline $\mathrm{Al}$ & - & 605 & 3.7 & Al-droplet $0.9 \mathrm{~nm}$ plus $1 \mathrm{ML} \mathrm{Ala}$ \\
\hline $\mathrm{Al}_{0.33} \mathrm{Ga}_{0.67} \mathrm{As}$ & 10 & 590 & 33.7 & Tunnel barrier (high Al) \\
\hline $\mathrm{Al}_{0.15} \mathrm{Ga}_{0.85} \mathrm{As}$ & 15 & 590 & 64.1 & Tunnel barrier (low $\mathrm{Al}$ ) \\
\hline $\mathrm{Al}_{0.15} \mathrm{Ga}_{0.85} \mathrm{As}$ & 5 & 575 & 21.4 & Tunnel barrier (low-temperature) \\
\hline $\mathrm{Al}_{0.15} \mathrm{Ga}_{0.85} \mathrm{As}: \mathrm{Si}$ & 150 & 590 & 640.8 & $\mathrm{n}^{+}$-doped back gate \\
\hline $\mathrm{Al}_{0.15} \mathrm{Ga}_{0.85} \mathrm{As}$ & 50 & 590 & 209.3 & Buffer layer \\
\hline $\mathrm{AlAs} / \mathrm{Al}_{0.33} \mathrm{Ga}_{0.67} \mathrm{As}$ & $10 \times(67.08 / 59.54)$ & 590 & 8904.7 & Distributed Bragg reflector \\
\hline GalAs/AlAs & $22 \times(2.8 / 2.8)$ & 590 & 1101.7 & Short-period superlattice \\
\hline $\mathrm{GaAs}$ & 100 & 590 & 601.8 & Start \\
\hline
\end{tabular}

aFor the Al-layer, the amount of deposited aluminium is given as the thickness of a corresponding AlAs-layer. The aluminium is deposited in an arsenic-depleted ambience.

$\mathrm{b}$ In the molecular beam epitaxy chamber used here, the background impurity concentration is estimated to be $\sim 5 \times 10^{14} \mathrm{~cm}^{-3}$ for $\mathrm{Al}_{0.33} \mathrm{Ga}_{0.67} \mathrm{As}$ layers ${ }^{50}$. The doping concentration is around $2 \times 10^{18} \mathrm{~cm}^{-3}$ for the $\mathrm{n}^{+}$layer, while for $\mathrm{p}^{+}$and $\mathrm{p}^{++}$layers, it is around $2 \times 10^{18} \mathrm{~cm}^{-3}$ and $8 \times 10^{18} \mathrm{~cm}^{-3}$, respectively. Between the $n$-type back gate and the $p$-type top gate, the sample has a built-in potential of $1.82 \mathrm{~V}$.

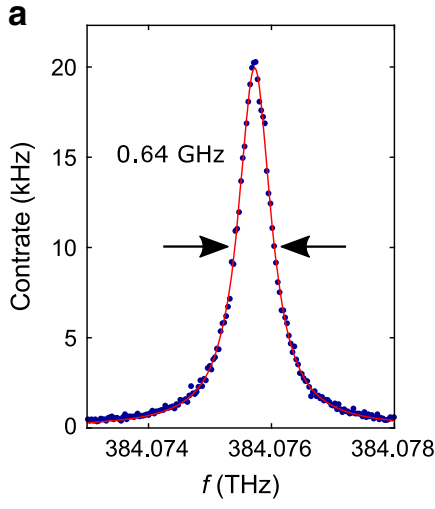

e

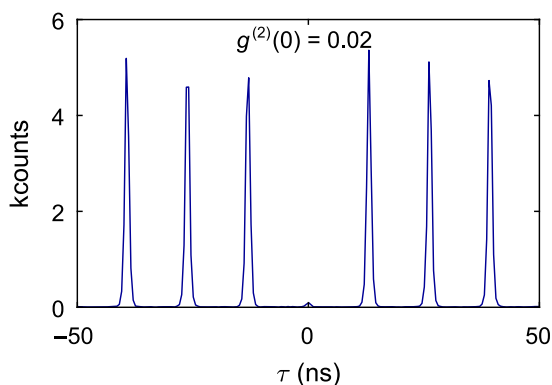

b

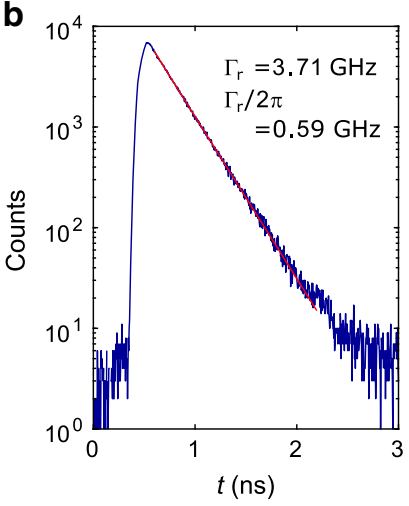

$\mathbf{f}$

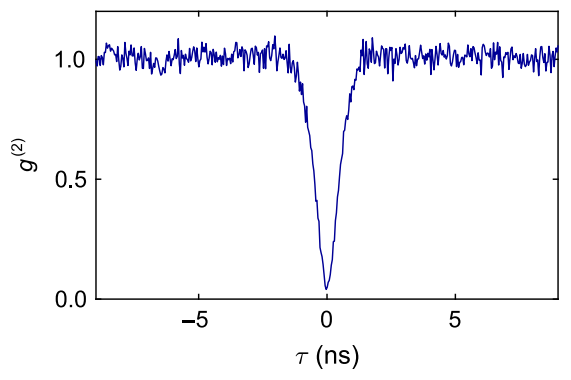

C

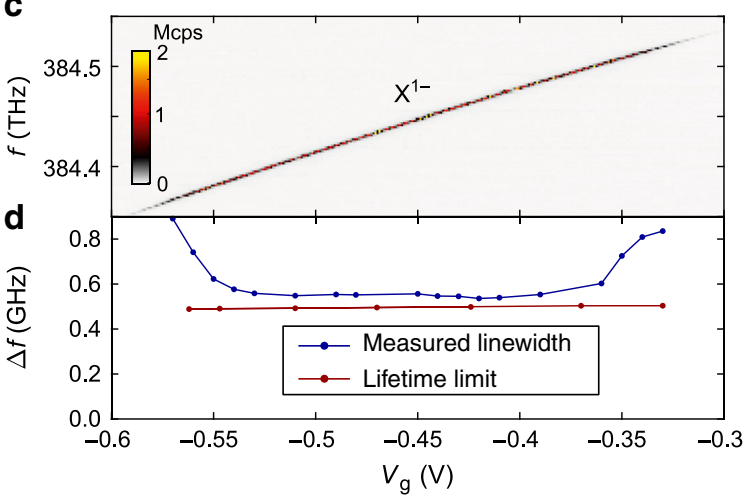

g

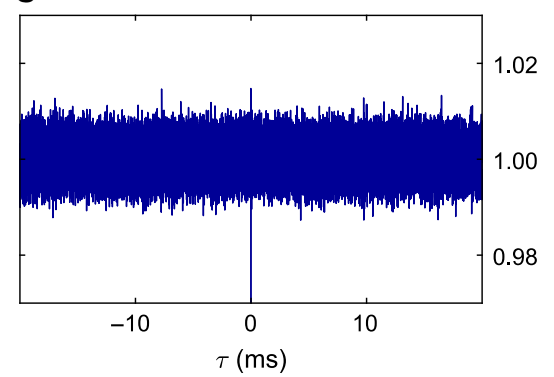

Fig. 2 Time-resolved lifetime and photon-correlation measurements. a Resonance fluorescence linewidth measured on the singly-charged exciton, $X^{1-}$ (QD1). The measurement is performed by sweeping a narrow-bandwidth laser over the $X^{1-}$ resonance. The overall time for the shown scan is $\sim 8$ min. A Lorentzian function (red line) fits perfectly to the data (blue dots), showing an optical linewidth of $0.64 \pm 0.01 \mathrm{GHz}$. b Lifetime measurement on $\mathrm{X}^{1-}$ under pulsed resonant excitation. The gate voltage is the same as in $\mathbf{a}$. The measured decay rate $\left(\Gamma_{\mathrm{r}}=3.71 \pm 0.04 \mathrm{GHz}\right.$, corresponding to a lifetime of $1 / \Gamma_{\mathrm{r}}=270 \pm 3 \mathrm{ps}$ ) implies a lifetime-limited linewidth of $\Gamma_{\mathrm{r}} / 2 \pi=0.59 \pm 0.01 \mathrm{GHz}$ (Exponential fit). c Resonance fluorescence of $X^{1-}$ (QD2) as a function of the gate voltage. $\mathbf{d}$ Resonance fluorescence linewidth along with the lifetime-limit (obtained from separate lifetime measurements at the corresponding gate voltages). Similar to QD1, the linewidth of QD2 stays very close to the lifetime limit in the plateau centre. e Auto-correlation ( $g^{(2)}$ ) measured under resonant $\pi$-pulse excitation. $\mathbf{f}$ Auto-correlation of the resonance fluorescence measured under weak continuous-wave excitation shown on a short timescale. The $g^{(2)}$-measurement is normalised ${ }^{44}$ by dividing the number of coincidences by its expectation value $T \cdot t_{\text {bin }} \cdot x_{1} \cdot x_{2}$, where $T$ is the overall integration time, $t_{\text {bin }}$ is the binning time, and $x_{1}, x_{2}$ are the count-rates on the two single-photon detectors. $\mathbf{g}$ The same auto-correlation measurement as in f but evaluated on a much longer time-scale (milliseconds). The perfectly flat $g^{(2)}$ reveals the absence of blinking.

A remarkable feature is that the close-to-transform limited linewidths are observed despite the large dc Stark shifts of these QDs. Within the $\mathrm{X}^{1-}$ plateau of QD1 (Fig. 1c), the dc Stark shift is $0.0347 \mathrm{GHz}$ per $\mathrm{V} \mathrm{cm}^{-1}$, about a factor of four larger than the typical dc Stark shifts of InGaAs QDs ${ }^{40}$. The sensitivity of the transition frequency to the electric field renders the QD linewidth susceptible to charge noise. The close-to-transform limited linewidths reflect therefore an extremely low level of charge 
noise in the device. Assuming that the slight increase in broadening with respect to the transform limit arises solely from charge noise, the linewidth measurement places an upper bound of $\sim 3.0 \mathrm{~V} \mathrm{~cm}^{-1}$ for the root-mean-square (rms) electric field noise at the location of QD1. This upper bound is comparable to the best gated InGaAs QD devices $20,29,40,42,43$.

For applications as single-photon source, it is crucial to demonstrate that the photons are emitted one by one, i.e., photon anti-bunching. Therefore, we continue our analysis by performing an intensity auto-correlation of the resonance fluorescence. This $g^{(2)}$-measurement is shown in Fig. 2e and Supplementary Fig. $6 \mathrm{c}, \mathrm{d}$ for resonant $\pi$-pulse excitation with $76 \mathrm{MHz}$ repetition rate. We observe a strong anti-bunching at zero time delay $\left(g^{(2)}\right.$ $(0)=0.019 \pm 0.008)$, corresponding to a single-photon purity of $1-g^{(2)}(0) \sim 98 \%$. The corresponding measurement under weak continuous-wave excitation is shown in Fig. $2 \mathrm{f}$. $\left(g^{(2)}\right.$-measurements versus excitation power, as well as laser detuning are mapped out in Supplementary Fig. 7, where clear Rabi oscillations are shown. In both cases, we find excellent agreement between the measured $g^{(2)}$ and a calculation based on a two-level model.) Also here, we observe a strong anti-bunching proving the singlephoton nature of the emission.

Previous resonance fluorescence on GaAs QDs has suffered from blinking, i.e., telegraph noise in the emission ${ }^{22}$. This is a deleterious consequence of charge noise: either the QD charges abruptly or the charge state of a nearby trap changes, detuning the QD from the excitation laser in both cases. Blinking gives rise to a characteristic bunching $\left(g^{(2)}>1\right)$ in the auto-correction even for driving powers well below saturation ${ }^{22}$. We investigate this point here. Even out to long (millisecond) time-scales, the $g^{(2)}$ measurement is absolutely flat and close to one (see Fig. $2 \mathrm{~g}$ ). (We note that our analysis includes a mathematically justified normalisation of the $g^{(2)}$-measurement $\left.{ }^{44}\right)$. This result demonstrates that blinking is absent. This is a consequence both of the diode-structure, in particular Coulomb blockade which locks the QD charge, and the low charge noise in the material surrounding the QD.

We subsequently carried out $g^{(2)}$-measurements with either a small magnetic field along the growth direction or a laser slightly detuned from the $\mathrm{QD}$ resonance. In the former case the sensitivity to spin noise is enhanced, while in the latter case the sensitivity to charge noise is enhanced ${ }^{40}$. In Supplementary Fig. 8, we compare the $g^{(2)}$-measurements on millisecond time-scales. For the measurement with an additional magnetic field (Supplementary Fig. 8c, d), the $g^{(2)}$ remains flat and stays close to one. In contrast, we observe a small blinking when the laser is detuned (Supplementary Fig. 8e, f). We infer from these results that in our device charge noise is most likely to be responsible for the residual linewidth broadening.

High-fidelity spin initialisation. The diode structure allows us to load a QD with a single electron. The spin of the electron is a valuable quantum resource. To probe the electron-spin dynamics, we probe the $\mathrm{X}^{1-}$ resonance fluorescence in a magnetic field (Faraday-geometry). In this configuration, the ground state is split by the electron Zeeman energy, and the excited state is split by the hole Zeeman energy (see Fig. 3a). As the diagonal transitions in this level-scheme are close to forbidden, the $\mathrm{X}^{1-}$. charge-plateau splits into two lines which are separated by the sum of electron and hole Zeeman energies (see Fig. 3b). We find that the $\mathrm{X}^{1-}$ charge-plateau becomes optically dim in its centre. This is the characteristic feature of spin-initialisation via optical pumping $27,43,45,46$. On driving e.g., the $|\uparrow\rangle-|\uparrow \downarrow \uparrow\rangle$ transition, the trion will most likely decay back to the $|\uparrow\rangle$-state via the dipoleallowed vertical transition. However, due to the heavy-hole

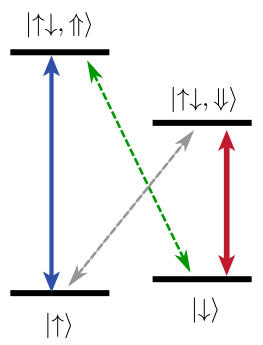

b

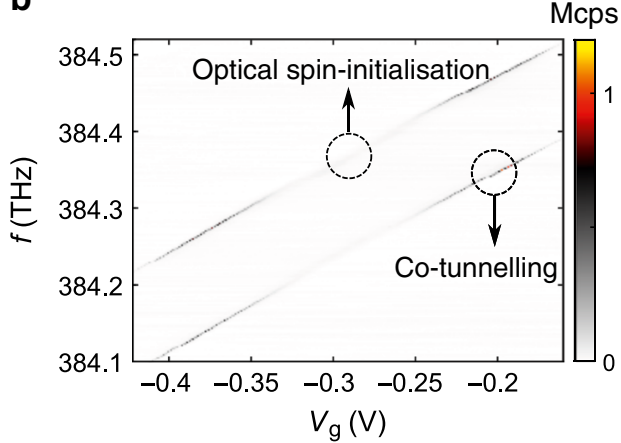

e

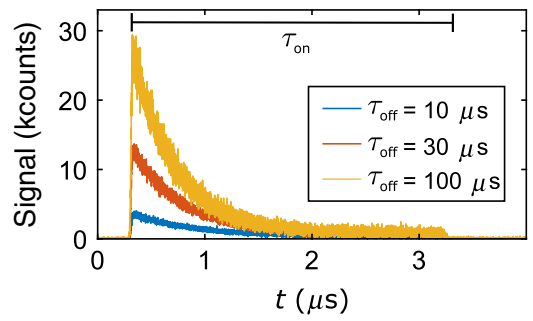

C

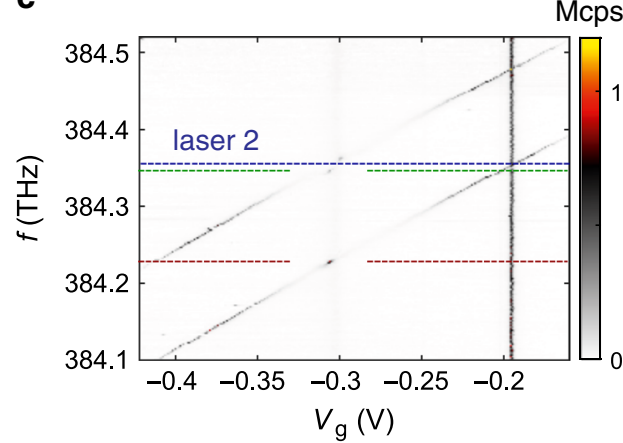

f

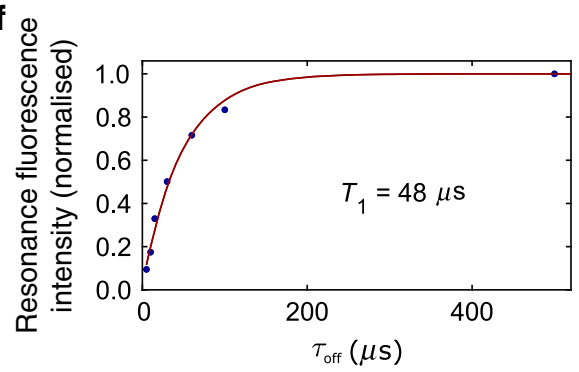

Fig. 3 Initialisation of a single electron spin. a Level scheme of the negative trion $X^{1-}$ in a magnetic field (Faraday geometry). b Optical spin-initialisation via optical pumping on $\mathrm{X}^{1-}$. The measurement is carried out at $B=6.6 \mathrm{~T}$. In the plateau centre, the resonance fluorescence disappears due to successful spin-initialisation; at the plateau edges it remains bright due to rapid spin-randomisation via co-tunnelling ${ }^{41}$. c Optical spin-initialisation and re-pumping with a second laser at a fixed frequency (laser 2). Recoveries of the signal are found in the plateau centre. $\mathbf{d}$ Schematic of the time-resolved spin-pumping measurement. e Resonance fluorescence intensity as a function of time. The signal drops due to optical spin-initialisation after turning the driving laser on. The overall intensity is larger when the time-delay $\tau_{\text {off }}$ between the laser pulses is larger. In this case, the electron spin has more time to relax back from the off-resonant state. $\mathbf{f}$ Resonance fluorescence intensity as a function of the waiting time between the spin-pumping laser pulses. The magenta line is an exponential fit to the data (blue dots). From this measurement we extract an electron-spin lifetime of $T_{1} \sim 48 \pm 5 \mu \mathrm{s}$. 
light-hole mixing or a weak in-plane nuclear field, it can also decay to the $|\downarrow\rangle$-state through the "forbidden" transtion with a small probability. When the QD is in the $|\downarrow\rangle$-state, the driving laser is off-resonance on account of the electron Zeeman energy. Therefore, the centre of the $\mathrm{X}^{1-}$-charge-plateau becomes dark and the initialisation of the electron spin in the $|\downarrow\rangle$-state is heralded by the disappearing resonance fluorescence. At the plateauedges, resonance fluorescence reappears due to fast spinrandomisation via co-tunnelling ${ }^{41}$. By comparing the remaining intensity in the charge-plateau centre to the plateau edges ${ }^{27}$, we estimate the spin initialisation fidelity to be $F=98.3 \pm 0.3 \%$. To confirm that the signal disappears in the plateau-centre on account of optical spin initialisation and not some other process, we perform a measurement with a second laser at a fixed frequency. When the fixed laser is resonant with $|\uparrow\rangle-|\uparrow \downarrow \uparrow\rangle$ transition, we observe a recovery of the signal (Fig. 3c) on either driving the weak diagonal transition $|\downarrow\rangle-|\uparrow \downarrow \uparrow\rangle$ or the strong vertical transitions $|\downarrow\rangle-|\uparrow \downarrow \Downarrow\rangle$ with the scan laser. While the fixed laser is tuned to $|\downarrow\rangle-|\uparrow \downarrow \uparrow\rangle$ transition (at a different $V_{\mathrm{g}}$ ), another recovery spot is seen as the scan laser drives the vertical transition $|\uparrow\rangle-|\uparrow \downarrow \uparrow\rangle$. This confirms the optical spininitialisation mechanism ${ }^{27,45}$. From the energy splitting at the plateau edges, we determine the electron and hole g-factors ${ }^{17}$, $g_{\mathrm{e}}=-0.076 \pm 0.001$ and $g_{\mathrm{h}}=1.309 \pm 0.001$. For the positively charged trion $\left(\mathrm{X}^{1+}\right)$, we also observe high-fidelity optical spininitialisation (Supplementary Fig. 9) and narrow linewidths (0.62 $\mathrm{GHz}$, see Supplementary Fig. 3), in this case of a hole spin.

How long-lived is the prepared spin state? To answer this question, we measure the time-dependence of the $\mathrm{X}^{1-}$ spin initialisation $^{43,46}$. The scheme is illustrated in Fig. 3d. First, we drive the $|\uparrow\rangle-|\uparrow \downarrow \uparrow\rangle$ transition for $\tau_{\text {on }}=3 \mu$ s. During this laser pulse, the signal decreases due to optical spin-initialisation (Fig. 3e). Subsequently, we turn the laser off for a time $\tau_{\text {off }}$, and then turn the laser back on again. During the off-time the electron spin randomises. Fig. $3 \mathrm{e}$ shows that the resonance fluorescence signal is stronger when the waiting time $\tau_{\text {off }}$ is longer. The reason for this effect is that with increasing $\tau_{\text {off }}$ the spin has more time to randomise. For a short value of $\tau_{\text {off, }}$ in contrast, the spin remains in the off-resonant state-it has no time to relax before the next optical pulse is applied. By measuring the signal strength for varying $\tau_{\text {off }}$ (Fig. $3 \mathrm{f}$ ), we determine an electron-spin relaxation time of $T_{1}=48 \pm 5 \mu \mathrm{s}$. Our result shows that the design of the tunnel-barrier between QDs and back gate is well suited for spinexperiments on single QDs. This $T_{1}$ value is significantly larger compared to the GaAs QDs without the $n$ - $i$ - $p$-diode structure ${ }^{23}$. The point is that the $T_{1}$ time is potentially longer than the coherence time $T_{2}$, such that the relaxation process governing $T_{1}$ is unlikely to limit the coherence time $T_{2}{ }^{47}$.

\section{Discussion}

In summary, we have developed charge-tunable GaAs QDs with ultra-low charge noise. We show notable improvements of the GaAs QDs properties: optical linewidths are close-to lifetimelimited, blinking is eliminated, and long electron-spin lifetimes are achieved. From a materials perspective, the crucial advance is the new diode structure hosting GaAs QDs-a key feature is that all the doping is incorporated in layers of low Al-concentration. In this way, the occupation of DX-centres is avoided and the AlGaAs layers are conducting at low temperatures. The concepts developed in this work can be transferred to thinner diodestructures that allow integration into photonic-crystals and other nanophotonic devices 3,36 . From a quantum photonics perspective, our results pave the way to bright sources of low-noise single photons close to the red part of the visible spectrum. This will facilitate the developments of both short-range networks and a hybrid QD-rubidium quantum memory. On account of the lowstrain environment in GaAs QDs, our work can also open the door to prolonged electron spin coherence.

\section{Methods}

Sample fabrication. The sample heterostructure and the quantum dots are grown by molecular beam epitaxy (MBE). The MBE setup is similar to the one described in ref. ${ }^{48}$. The complete heterostructure of the sample is shown in Table 1. All doped layers in AlGaAs have low Al-concentration $(<20 \%)$. The quantum dots are surrounded by AlGaAs with higher Al-concentration (33\%), to enable the growth of QDs close to rubidium-frequencies and with small fine-structure splittings ${ }^{8,11}$. We fabricate separate Ohmic contacts to the $\mathrm{n}^{+}$and $\mathrm{p}^{++}$layers. For the $n$-type back gate, the sample is locally etched down by $\sim 360 \mathrm{~nm}$ in a mixture of sulfuric acid and hydrogen peroxide (concentrated $\mathrm{H}_{2} \mathrm{SO}_{4}: 30 \% \mathrm{H}_{2} \mathrm{O}_{2}: \mathrm{H}_{2} \mathrm{O}=1: 1: 50$ ). $\mathrm{NiAuGe}$ is then deposited by electron-beam evaporation (with three steps: $60 \mathrm{~nm}$ AuGe (mass ratio 88:12), $10 \mathrm{~nm} \mathrm{Ni}$, and $60 \mathrm{~nm} \mathrm{AuGe}$ ), followed by thermal annealing at $370{ }^{\circ} \mathrm{C}$ for $60 \mathrm{~s}$ and $420^{\circ} \mathrm{C}$ for $30 \mathrm{~s}$. For the $p$-type top gate, a thin contact pad consisting of $\mathrm{Ti}(3 \mathrm{~nm}) / \mathrm{Au}(7 \mathrm{~nm})$ is evaporated locally on the top surface of the sample. Both contacts are electrically connected with silver paint.

Experimental setups. The sample is cooled down to $4.2 \mathrm{~K}$ in a liquid helium cryostat. We perform photoluminescence with a $632.8 \mathrm{~nm} \mathrm{He}-\mathrm{Ne}$ laser. The photoluminescence is collected by an aspheric objective lens (numerical aperture $\mathrm{NA}=0.71$ ) and sent to a spectrometer. Resonance fluorescence is performed with a narrow-band laser (1 MHz linewidth), using a cross-polarisation confocal darkfield microscope 22,49 to distinguish QD-signal from the scattered laser light. It is detected using superconducting-nanowire single-photon detectors and a counting hardware with a total timing jitter of $\sim 35$ ps (full width at half maximum).

Statistics of QD linewidths. In our device, GaAs QDs with a small height (emission wavelength below $\sim 785 \mathrm{~nm}$ ) tend to have excellent optical properties. We find that more than every second QD has a close to lifetime-limited linewidth (see Supplementary Fig. 4a,b). This includes QDs close to the ${ }^{87} \mathrm{Rb}_{2}$ line ( 780 $\mathrm{nm}$ ). For QDs larger in size (emission wavelength above $\sim 785 \mathrm{~nm}$ ), the QD linewidths are usually broader. The reason is probably the following: the GaAs QDs in our sample are grown by infilling nano-holes droplet-etched into a $10 \mathrm{~nm}$-thin layer of $\mathrm{Al}_{0.33} \mathrm{Ga}_{0.67} \mathrm{As}$ (see Table 1). The depths of the nano-holes, and therefore the heights of the QDs, typically range from $5 \mathrm{~nm}$ to $10 \mathrm{~nm}^{8,11}$. A QD emitting at higher wavelength tends to have a larger height ${ }^{11}$. When the height of a QD comes close to $10 \mathrm{~nm}$, the optical properties could be affected by the $\mathrm{Al}_{0.33} \mathrm{Ga}_{0.67} \mathrm{As} /$ $\mathrm{Al}_{0.15} \mathrm{Ga}_{0.85}$ As interface. A simple solution is to make the $\mathrm{Al}_{0.33} \mathrm{Ga}_{0.67} \mathrm{As}$-layer $5 \mathrm{~nm}$ thicker. In this case, we expect good optical properties also for QDs of higher wavelengths.

Auto-correlation under different excitation schemes. We investigate the stability of the QD under different excitation schemes. We start with continuous-wave (CW) excitation. We perform auto-correlation measurements on $\mathrm{X}^{1-}$ at a constant gate voltage while exciting the QD with (i) an above-band laser $(\lambda=632.8 \mathrm{~nm}$ ), (ii) a laser resonant with the $p$-shell, and (iii) a laser resonant with the $s$-to-s transition The results are shown in (i) Supplementary Fig. 6a, (ii) Supplementary Fig. 6b, and (iii) Fig. 2 g, respectively. In all three cases, the $g^{(2)}$ stays very flat and close to onethere is no blinking even on a long time-scale. This shows that the QD is a very stable quantum emitter under all three CW excitation schemes. From an applications point of view, it is usually necessary to drive the QD with a resonant pulsed laser. We investigate the auto-correlation under resonant $\pi$-pulse excitation in Fig. 2e. An evaluation of this $g^{(2)}$-measurement on a longer time-scale is plotted in Supplementary Fig. $6 c$, where the $y$-axis is displayed on a logarithmic scale to resolve the central peak. To investigate whether a strong $\pi$-pulse introduces any blinking, we plot the $g^{(2)}$-measurement in a histogram plot (Supplementary Fig. 6d) by summing up the coincidence events for every single pulse. This sum is divided by the expectation value for a perfectly stable source: the normalisation factor is $x_{1} x_{2} T_{\text {int }} / f_{\text {rep }}$, where $f_{\text {rep }}$ is the repetition rate of the pulsed laser, $x_{1}, x_{2}$ represent the count rates of the two detectors used for a $T_{\text {int }}$-long $g^{(2)}$-measurement. A derivation of the normalisation factor is given in Supplementary Fig. 6. Importantly, the histogram bars at non-zero time delay are flat and very close to one; the bar at zero delay is close to zero. This shows that the QD is a stable single-photon emitter for resonant $\pi$-pulse excitation.

Potential noise source affecting the QD-linewidth. The $g^{(2)}$-measurement shown in Fig. 2f,g is performed on a trion at zero magnetic field when the CW laser drives the QD resonantly. The sensitivity can be enhanced towards either spin noise or charge noise by applying a small magnetic field along the growth direction, and detuning the laser slightly from the QD-resonance by $\delta$, respectively. A trion state is degenerate at zero magnetic field, consisting of two opposite spin ground states. When applying a magnetic field $B$, the degeneracy is lifted and the trion state is split into two by a Zeeman energy $E_{z}=g \mu_{B} B$, with $g$ being the electron or hole $g$ factor, and $\mu_{B}$ the Bohr magneton. We maximise the spin noise sensitivity by applying a small magnetic field such that $E_{z}=\frac{\tilde{\Gamma}}{\sqrt{3}}$ (Supplementary Fig. 8c). Here $\tilde{\Gamma}$ 
represents the full width at half maximum (FWHM) of the QD emission. For the maximised spin noise sensitivity, the $g^{(2)}$-measurement does not show any clear sign of bunching (Supplementary Fig. 8d). The charge noise sensitivity is maximised when the laser is detuned from the QD by $\delta=\frac{\tilde{\Gamma}}{2 \sqrt{3}}$ (Supplementary Fig. 8e). In this configuration, we observe a small bunching peak in the $g^{(2)}$-measurement (Supplementary Fig. 8f). This result suggests that charge noise on a millisecond time-scale is responsible for the slight linewidth broadening.

\section{Data availability}

The data that supports this work is available from the corresponding author upon reasonable request.

\section{Code availability}

The code that has been used for this work is available from the corresponding author upon reasonable request.

Received: 25 March 2020; Accepted: 3 September 2020;

Published online: 21 September 2020

\section{References}

1. Somaschi, N. et al. Near-optimal single-photon sources in the solid state. Nat. Photon. 10, 340-345 (2016).

2. Ding, X. et al. On-demand single photons with high extraction efficiency and near-unity indistinguishability from a resonantly driven quantum dot in a micropillar. Phys. Rev. Lett. 116, 020401 (2016).

3. Liu, F. et al. High Purcell factor generation of indistinguishable on-chip single photons. Nat. Nanotechnol. 13, 835 (2018).

4. Liu, J. et al. A solid-state source of strongly entangled photon pairs with high brightness and indistinguishability. Nat. Nanotechnol. 14, 586-593 (2019).

5. Gao, W. B., Fallahi, P., Togan, E., Miguel-Sanchez, J. \& Imamoglu, A. Observation of entanglement between a quantum dot spin and a single photon. Nature 491, 426-430 (2012).

6. Stockill, R. et al. Phase-tuned entangled state generation between distant spin qubits. Phys. Rev. Lett. 119, 010503 (2017).

7. Sangouard, N., Simon, C., de Riedmatten, H. \& Gisin, N. Quantum repeaters based on atomic ensembles and linear optics. Rev. Mod. Phys. 83, 33-80 (2011).

8. Huo, Y. H., Rastelli, A. \& Schmidt, O. G. Ultra-small excitonic fine structure splitting in highly symmetric quantum dots on GaAs (001) substrate. Appl. Phys. Lett. 102, 152105 (2013).

9. Gurioli, M., Wang, Z., Rastelli, A., Kuroda, T. \& Sanguinetti, S. Droplet epitaxy of semiconductor nanostructures for quantum photonic devices. Nat. Mater. 18, 799-810 (2019)

10. Heyn, C. et al. Highly uniform and strain-free GaAs quantum dots fabricated by filling of self-assembled nanoholes. Appl. Phys. Lett. 94, 183113 (2009).

11. Löbl, M. C. et al. Correlations between optical properties and Voronoi-cell area of quantum dots. Phys. Rev. B 100, 155402 (2019).

12. Wolters, J. et al. Simple atomic quantum memory suitable for semiconductor quantum dot single photons. Phys. Rev. Lett. 119, 060502 (2017).

13. Basso Basset, F. et al. High-yield fabrication of entangled photon emitters for hybrid quantum networking using high-temperature droplet epitaxy. Nano Lett. 18, 505-512 (2018).

14. Urbaszek, B. et al. Nuclear spin physics in quantum dots: an optical investigation. Rev. Mod. Phys. 85, 79-133 (2013).

15. Plumhof, J. D. et al. Experimental investigation and modeling of the fine structure splitting of neutral excitons in strain-free $\mathrm{GaAs} / \mathrm{Al}_{x} \mathrm{Ga}_{1-x} \mathrm{As}$ quantum dots. Phys. Rev. B 81, 121309(R) (2010).

16. Ha, N. et al. Size-dependent line broadening in the emission spectra of single GaAs quantum dots: Impact of surface charge on spectral diffusion. Phys. Rev. B 92, 075306 (2015).

17. Ulhaq, A. et al. Vanishing electron $g$ factor and long-lived nuclear spin polarization in weakly strained nanohole-filled GaAs/AlGaAs quantum dots. Phys. Rev. B 93, 165306 (2016).

18. Gangloff, D. et al. Quantum interface of an electron and a nuclear ensemble. Science 364, 62-66 (2019).

19. Shulman, M. D. et al. Suppressing qubit dephasing using real-time hamiltonian estimation. Nat. Commun. 5, 5156 (2014).

20. Najer, D. et al. A gated quantum dot strongly coupled to an optical microcavity. Nature 575, 622-627 (2019).

21. Schwartz, I. et al. Deterministic generation of a cluster state of entangled photons. Science 354, 434-437 (2016).

22. Jahn, J.-P. et al. An artificial $\mathrm{Rb}$ atom in a semiconductor with lifetime-limited linewidth. Phys. Rev. B 92, 245439 (2015).
23. Béguin, L. et al. On-demand semiconductor source of 780-nm single photons with controlled temporal wave packets. Phys. Rev. B 97, 205304 (2018).

24. Kumar, S. et al. Strain-induced tuning of the emission wavelength of high quality GaAs/AlGaAs quantum dots in the spectral range of the ${ }^{87} \mathrm{Rb} \mathrm{D}_{2}$ lines. Appl. Phys. Lett. 99, 161118 (2011).

25. Schöll, E. et al. Resonance fluorescence of GaAs quantum dots with near-unity photon indistinguishability. Nano Lett. 19, 2404-2410 (2019).

26. Warburton, R. J. et al. Optical emission from a charge-tunable quantum ring Nature 405, 926-929 (2000)

27. Löbl, M. C. et al. Narrow optical linewidths and spin pumping on chargetunable close-to-surface self-assembled quantum dots in an ultrathin diode. Phys. Rev. B 96, 165440 (2017).

28. Grim, J. Q. et al. Scalable in operando strain tuning in nanophotonic waveguides enabling three-quantum-dot superradiance. Nat. Mater. 18, 963-969 (2019).

29. Kuhlmann, A. V. et al. Transform-limited single photons from a single quantum dot. Nat. Commun. 6, 8204 (2014).

30. Patel, R. B. et al. Two-photon interference of the emission from electrically tunable remote quantum dots. Nat. Photon. 4, 632-635 (2010).

31. Langer, F., Plischke, D., Kamp, M. \& Höfling, S. Single photon emission of a charge-tunable GaAs/ $/ \mathrm{Al}_{0.25} \mathrm{Ga}_{0.75} \mathrm{As}$ droplet quantum dot device. Appl. Phys. Lett. 105, 081111 (2014).

32. Bouet, L. et al. Charge tuning in [111] grown gaas droplet quantum dots. Appl. Phys. Lett. 105, 082111 (2014).

33. Mooney, P. Deep donor levels (DX centers) in III-V semiconductors. J. Appl. Phys. 67, R1-R26 (1990).

34. Munoz, E. et al. Techniques to minimize DX center deleterious effects in III-V device performance. J. Appl. Phys. 73, 4988-4997 (1993).

35. Böckler, C. et al. Electrically driven high-Q quantum dot-micropillar cavities. Appl. Phys. Lett. 92, 091107 (2008).

36. Kiršanské, G. et al. Indistinguishable and efficient single photons from a quantum dot in a planar nanobeam waveguide. Phys. Rev. B 96, 165306 (2017).

37. Fry, P. W. et al. Inverted electron-hole alignment in InAs-GaAs self-assembled quantum dots. Phys. Rev. Lett. 84, 733-736 (2000).

38. Huber, D. et al. Strain-tunable gaas quantum dot: A nearly dephasing-free source of entangled photon pairs on demand. Phys. Rev. Lett. 121, 033902 (2018).

39. Madsen, K. H. et al. Measuring the effective phonon density of states of a quantum dot in cavity quantum electrodynamics. Phys. Rev. B 88, 045316 (2013).

40. Kuhlmann, A. V. et al. Charge noise and spin noise in a semiconductor quantum device. Nat. Phys. 9, 570-575 (2013).

41. Smith, J. M. et al. Voltage control of the spin dynamics of an exciton in a semiconductor quantum dot. Phys. Rev. Lett. 94, 197402 (2005).

42. Matthiesen, C., Vamivakas, A. N. \& Atatüre, M. Subnatural linewidth single photons from a quantum dot. Phys. Rev. Lett. 108, 093602 (2012).

43. Lu, C.-Y. et al. Direct measurement of spin dynamics in InAs/GaAs quantum dots using time-resolved resonance fluorescence. Phys. Rev. B 81, 035332 (2010).

44. Löbl, M. C. et al. Radiative Auger process in the single-photon limit. Nat. Nanotechnol. 15, 558-562 (2020).

45. Atatüre, M. et al. Quantum-dot spin-state preparation with near-unity fidelity. Science 312, 551-553 (2006)

46. Javadi, A. et al. Spin-photon interface and spin-controlled photon switching in a nanobeam waveguide. Nat. Nanotechnol. 13, 398 (2018).

47. Stockill, R. et al. Quantum dot spin coherence governed by a strained nuclear environment. Nat. Commun. 7, 12745 (2016).

48. Ludwig, A. et al. Ultra-low charge and spin noise in self-assembled quantum dots. J. Cryst. Growth 477, 193-196 (2017).

49. Kuhlmann, A. V. et al. A dark-field microscope for background-free detection of resonance fluorescence from single semiconductor quantum dots operating in a set-and-forget mode. Rev. Sci. Instrum. 84, 073905 (2013).

50. MacLeod, S. J. et al. Hybrid architecture for shallow accumulation mode $\mathrm{AlGaAs} / \mathrm{GaAs}$ heterostructures with epitaxial gates. Appl. Phys. Lett. 106, 012105 (2015)

\section{Acknowledgements}

The authors thank Jan-Philipp Jahn and Armando Rastelli for stimulating discussions. L.Z. received funding from the European Union's Horizon 2020 Research and Innovation programme under the Marie Skłodowska-Curie grant agreement No. 721394 (4PHOTON). M.C.L., C.S., and R.J.W. acknowledge financial support from NCCR QSIT and from SNF Project No. 200020_156637. J.R., A.L., and A.D.W. gratefully acknowledge financial support from the grants DFH/UFA CDFA05-06, DFG TRR160, DFG project 383065199, and BMBF Q.Link.X 16KIS0867. A.J. acknowledge support from the European Union's Horizon 2020 research and innovation programme under the Marie Skłodowska-Curie grant agreement No. 840453 (HiFig). 


\section{Author contributions}

L.Z., M.C.L., G.N.N., A.J., and C.S. carried out the experiments. L.Z., M.C.L., J.R., and A.L. designed the sample. J.R., L.Z., A.D.W., and A.L. grew the sample. C.S., L.Z., G.N.N., and M.C.L. fabricated the sample. L.Z., M.C.L., C.S., G.N.N., A.J., and R.J.W. analysed the data. M.C.L., L.Z., and R.J.W. wrote the manuscript with input from all the authors.

\section{Competing interests}

The authors declare no competing interests.

\section{Additional information}

Supplementary information is available for this paper at https://doi.org/10.1038/s41467020-18625-z.

Correspondence and requests for materials should be addressed to L.Z.

Peer review information Nature Communications thanks the anonymous reviewer(s) for their contribution to the peer review of this work. Peer reviewer reports are available.
Reprints and permission information is available at http://www.nature.com/reprints

Publisher's note Springer Nature remains neutral with regard to jurisdictional claims in published maps and institutional affiliations.

\section{(c) (i)}

Open Access This article is licensed under a Creative Commons Attribution 4.0 International License, which permits use, sharing, adaptation, distribution and reproduction in any medium or format, as long as you give appropriate credit to the original author(s) and the source, provide a link to the Creative Commons license, and indicate if changes were made. The images or other third party material in this article are included in the article's Creative Commons license, unless indicated otherwise in a credit line to the material. If material is not included in the article's Creative Commons license and your intended use is not permitted by statutory regulation or exceeds the permitted use, you will need to obtain permission directly from the copyright holder. To view a copy of this license, visit http://creativecommons.org/licenses/by/4.0/.

(C) The Author(s) 2020 